\title{
A kinematic and incremental integration model for the micromechanical numerical analysis of dual-phase materials
}

\author{
F. Teixeira-Dias ${ }^{a, *}$, L.F. Menezes ${ }^{b}$ \\ a Departamento de Engenharia Mecânica, Universidade de Aveiro, Campus Santiago, 3810-193 Aveiro, Portugal \\ ${ }^{\mathrm{b}}$ Departamento de Engenharia Mecânica, Universidade de Coimbra, Polo II-Pinhal Marrocos, 3030 Coimbra, Portugal
}

\begin{abstract}
A kinematic model suitable for the numerical analysis of dual-phase materials and, in particular, for composite materials is presented. Due to the non-linear character of the behavior of most materials, the model must be presented in an incremental form. The kinematic model is based on the decomposition of the transformation gradient in thermoelastic and plastic parts. Thus, the velocity gradient tensor is also decomposed in an elastic and a plastic part. Two different constitutive models are implemented in order to test the model. The dual-phase material modeled is an $\mathrm{Al}-\mathrm{SiC}$ metal matrix composite (MMC). The reinforcement constitutive model is chosen to be thermoelastic and the matrix model is thermoelastic-viscoplastic. A forward gradient time integration procedure is described and implemented in order to calculate the increments of the state variables, namely the plastic strain increment and the stress increments in both materials. The proposed model is tested with one three-dimensional example: a cylindrical fiber MMC with $11 \%$ reinforcement volume fraction. The numerical results are compared with results obtained by other authors. Very good accordance can be observed between the results obtained with the incremental model proposed in this paper and those presented by other authors.
\end{abstract}

(C) 2002 Elsevier Science B.V. All rights reserved.

PACS: 81.05.-t; 81.05. Ni

Keywords: Dual-phase material; Incremental model; Kinematic approach; Integration; Micromechanics; Finite element method

\section{Introduction}

Dual-phase materials like, for example, metal matrix composites (MMCs), have become increasingly attractive in recent years for their particular mechanical and physical properties [1-9]. However, in the case of MMCs, there are often

\footnotetext{
${ }^{*}$ Corresponding author. Tel.: +351-234-370830; fax: $+351-$ 234-370953.

E-mail address: ftd@mec.ua.pt (F. Teixeira-Dias).
}

high levels of residual stresses generated when they are subjected to temperature variations. The development of such stresses and the mechanical behavior of dual-phase materials in the presence of these stresses have been thoroughly studied by several authors using analytical, numerical and/or experimental methods (e.g. [1-3,8-17]).

Some specific theoretical and numerical models have been proposed in order to evaluate these residual stresses. Both Arsenault and Taya [17] and Withers et al. [18] used Eshelby's equivalent inclusion approach to provide a theoretical basis for 
the prediction of the mechanical properties of short fiber MMCs. This approach allows the prediction of thermal residual stresses in MMCs considering thermoelastic fibers and plastic matrixes. Nevertheless, it has the drawback of being limited in the fields of the geometric and physical modelation of the problem. Dunn and Taya [19] also proposed an analysis of thermally induced residual stresses in dual-phase materials based on Eshelby's theory. The former studies the interaction among fibers at finite volume fractions through the Mori-Tanaka mean field theory and treats the matrix as an elastic-plastic material while the reinforcement is considered elastic. Povirk et al. [2] presented a general formulation valid for finite strains and rotations where account was taken of thermoelasticity in the reinforcement and of temperature-dependent plasticity in the matrix material. The flow theory of plasticity was used by Zahl et al. [9] to characterize a rateindependent ductile matrix while the reinforcement was considered to be both brittle and elastic. A mathematically more elaborate constitutive model was presented by Zywicz and Parks [13]. In this model the matrix material parameters are considered to be temperature dependent, including the coefficient of thermal expansion (CTE). The model used can describe a temperature- and time-dependent inelastic and isotropic matrix deformation, while the fiber reinforcements are modeled as linearly elastic and transversely isotropic. Suéry [10] and Teodosiu and Menezes [11] studied the residual stress fields in an aluminum matrix with a spherical $\mathrm{SiC}$ particle using a one-dimensional axisymmetric finite element code and considering a thermoelastic-viscoplastic behavior for the aluminum matrix and a thermoelastic constitutive model for the $\mathrm{SiC}$ reinforcement.

More recent papers have been published in this domain, but most of them use only one- or twodimensional models. This is an obvious limitation to the study of complex and more general reinforcement geometries and their influence on the resulting residual stress fields.

The purpose of the present paper is to present a three-dimensional micromechanical incremental model that is suitable to the numerical simulation of the behavior of dual-phase materials. The va- lidity of the proposed model is tested with one example concerning the cooling stage of a MMC after its fabrication process. The results are compared with those from other authors. For the particular case of the MMC materials, the mechanical model used considers that the reinforcement component behaves thermoelastically and the matrix material has a thermoelastic-viscoplastic behavior.

\section{Kinematics}

It is most important to follow the evolution of every material point when a dual-phase material is to be studied numerically. In order to follow the kinematics of a technological process, the authors propose using a Lagrangian updated integration formulation. It is thus necessary to determine the present configuration of the material, $C_{t}$, starting from the configuration of the previous instant, $C_{0}$, also known as reference configuration.

Let $P$ be a material point belonging to the solid body $\Omega$ and $\mathbf{p}$ and $\mathbf{x}$ the position vectors of the referred point in configurations $C_{0}$ and $C_{t}$, respectively. These configurations are schematically represented in Fig. 1. Thus

$\mathbf{x}=\overline{\mathbf{x}}(\mathbf{p}, t) \quad$ and $\quad \mathbf{p}=\overline{\mathbf{p}}(\mathbf{x}, t)$.

So, bearing in mind the previous definitions,

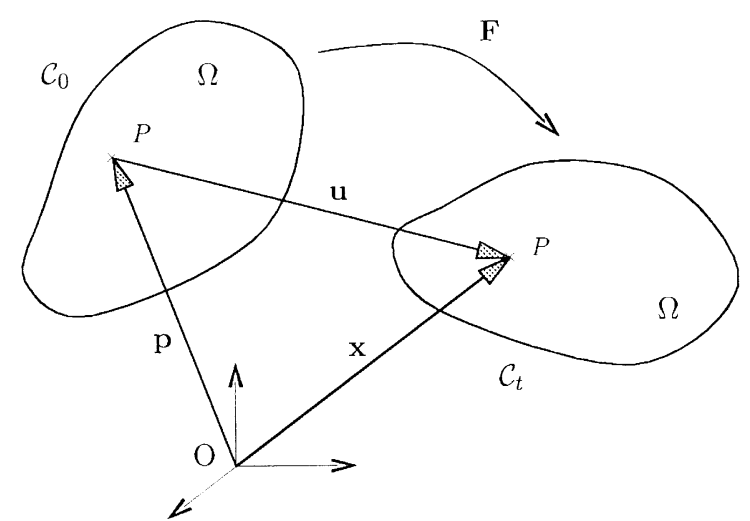

Fig. 1. Current configuration, $C_{t}$, and reference configuration, $C_{0}$, of the material at the initial and present time instants of the increment. 
$\mathbf{x}=\mathbf{p}+\mathbf{u}(\mathbf{p}, t)$,

where $\mathbf{u}(\mathbf{p}, t)$ is the total displacement of point $P$ between configurations $C_{0}$ and $C_{t}$. From relations (1) and (2) one can determine the transformation gradient $\mathbf{F}$ to be defined as:

$\mathbf{F}=\mathbf{F}(\mathbf{p}, t) \stackrel{\text { def }}{=} \frac{\partial}{\partial \mathbf{p}} \mathbf{u}(\mathbf{p}, t)$.

Inserting Eq. (2), the previous relation leads to

$\mathbf{F}=\mathbf{1}+\frac{\partial}{\partial \mathbf{p}} \mathbf{u}(\mathbf{p}, t)$

where $\mathbf{1}$ is the second order identity tensor. The velocity field associated to the previous transformation is

$\dot{\dot{\mathbf{x}}}(\mathbf{p}, t) \stackrel{\text { def }}{=} \frac{\partial}{\partial t} \overline{\mathbf{x}}(\mathbf{p}, t)$.

The spacial evolution of $\dot{\overline{\mathbf{x}}}(\mathbf{p}, t)$ can be described by

$\mathbf{v}(\mathbf{x}, t) \stackrel{\text { def }}{=} \dot{\overline{\mathbf{x}}}(\overline{\mathbf{p}}(\mathbf{x}, t), t)$

with the corresponding velocity gradient $\mathbf{L}$ defined as

$$
\begin{aligned}
\mathbf{L}(\mathbf{x}, t) \stackrel{\text { def }}{=} \operatorname{grad}[\mathbf{v}(\mathbf{x}, t)] & =\frac{\partial}{\partial \mathbf{x}} \mathbf{v}(\mathbf{x}, t) \\
& =\frac{\partial}{\partial \mathbf{p}} \dot{\overline{\mathbf{x}}}(\mathbf{p}, t) \frac{\partial}{\partial \mathbf{x}} \overline{\mathbf{p}}(\mathbf{x}, t),
\end{aligned}
$$

where grad is the gradient operator relative to $\mathbf{x}$ maintaining $t$ constant. With relation (3), Eq. (7) can be written as

$\mathbf{L}=\dot{\mathbf{F}} \mathbf{F}^{-1}$.

It is now necessary to separate the different behavior components in the transformation gradient $\mathbf{F}$ between time instants $t_{0}$ and $t$. For this purpose, it is considered that there are eventually three distinguishable behavior components: (i) elastic, (ii) plastic and (iii) thermal. The infinitesimal neighborhoods of material point $P$, in configurations $C_{0}$ and $C_{t}$ are schematically represented in Fig. 2. Additionally, consider the following virtual configurations: (i) $\Gamma_{0}$ - an infinitesimal region around $P$ at instant $t_{0}$ and temperature $T_{0}$; (ii) $\widehat{\Gamma}_{0}$ - configuration obtained after elastic relaxation of $\Gamma_{0}$. (iii) $\Gamma_{t}$ - an infinitesimal region around $P$ at

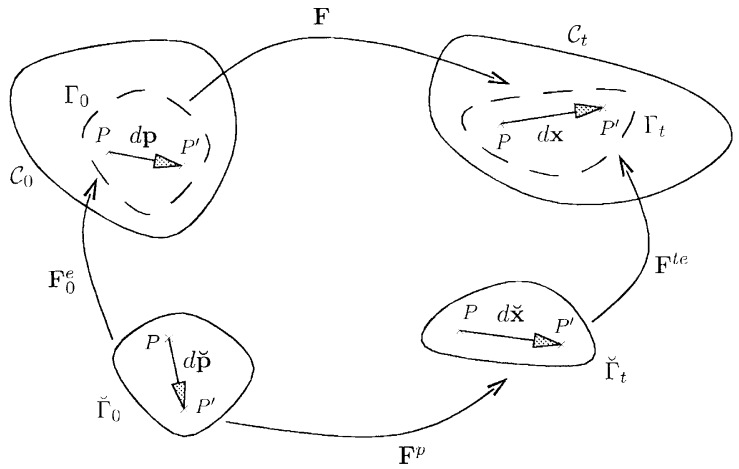

Fig. 2. Schematic representation of the transformation gradient decomposition for the incremental process.

instant $t$ and temperature $T$; (iv) $\widehat{\Gamma}_{t}$ - configuration obtained after elastic relaxation of $\Gamma_{t}$.

Let $\mathrm{d} \mathbf{p}$ be a position vector of a material point $P^{\prime}$, in an infinitesimal region around $P$, relative to $P$ itself. In this way, $\mathrm{d} \hat{\mathbf{p}}, \mathrm{d} \mathbf{x}$ and $\mathrm{d} \hat{\mathbf{x}}$ are the transformations of dp in configurations $\widehat{\Gamma}_{0}, \Gamma_{t}$ and $\widehat{\Gamma}_{t}$, respectively. With the previous definitions, three transformation gradients can be defined in the neighborhood of $P$ :

- $\mathbf{F}_{0}^{\mathrm{e}}$ - the transformation gradient between $\widehat{\Gamma}_{0}$ and $\Gamma_{0}$;

- $\mathbf{F}^{\mathrm{p}}$ - the transformation gradient between $\widehat{\Gamma}_{0}$ and $\widehat{\Gamma}_{t}$ and

- $\mathbf{F}^{\mathrm{te}}$ - the transformation gradient between $\widehat{\Gamma}_{t}$ and $\Gamma_{t}$.

It is now possible to relate vectors $\mathrm{dp}$ and $\mathrm{d} \mathbf{x}$ as

$\mathrm{d} \mathbf{x}=\mathbf{F}^{\mathrm{te}} \mathbf{F}^{\mathrm{p}}\left(\mathbf{F}_{0}^{\mathrm{e}}\right)^{-1} \mathrm{~d} \mathbf{p}$.

Inserting the definition of the transformation gradient $\mathbf{F}$ (Eq. (3)) relation (9) becomes

$\mathbf{F}=\mathbf{F}^{\mathrm{te}} \mathbf{F}^{\mathrm{p}}\left(\mathbf{F}_{0}^{e}\right)^{-1}$.

Replacing $\mathbf{F}$ in Eq. (8) leads to

$\mathbf{L}=\dot{\mathbf{F}}^{\mathrm{te}}\left(\mathbf{F}^{\mathrm{te}}\right)^{-1}+\mathbf{F}^{\mathrm{te}} \dot{\mathbf{F}}^{\mathrm{p}}\left(\mathbf{F}^{\mathrm{p}}\right)^{-1}\left(\mathbf{F}^{\mathrm{te}}\right)^{-1}$,

or

$\mathbf{L}=\mathbf{L}^{\mathrm{e}}+\mathbf{L}^{\mathrm{p}}$,

where $\mathbf{L}^{\mathrm{e}}=\dot{\mathbf{F}}^{\mathrm{te}}\left(\mathbf{F}^{\mathrm{te}}\right)^{-1}$ and $\mathbf{F}^{\mathrm{te}} \dot{\mathbf{F}}^{\mathrm{p}}\left(\mathbf{F}^{\mathrm{p}}\right)^{-1}\left(\mathbf{F}^{\mathrm{te}}\right)^{-1}$ are the elastic and plastic parts of the velocity gradient tensor $\mathbf{L}$, respectively. 
However, the velocity gradient tensor can still be decomposed in the strain rate tensor $\mathbf{D}$ and spin tensor $\mathbf{W}$ [1]

$\mathbf{L}=\mathbf{D}+\mathbf{W}$.

These tensors correspond to the symmetric $\left(\mathbf{L}^{\mathrm{S}}\right)$ and antisymmetric $\left(\mathbf{L}^{\mathrm{A}}\right)$ parts of $\mathbf{L}$, respectively, i.e.

$\mathbf{D}(\mathbf{x}, t) \stackrel{\text { def }}{=} \mathbf{L}^{\mathrm{S}}=\frac{1}{2}\left(\mathbf{L}+\mathbf{L}^{\mathrm{T}}\right)$

$\mathbf{W}(\mathbf{x}, t) \stackrel{\text { def }}{=} \mathbf{L}^{\mathrm{A}}=\frac{1}{2}\left(\mathbf{L}-\mathbf{L}^{\mathrm{T}}\right)$.

\section{Constitutive model}

A set of two constitutive models is used in the present work to describe the micromechanical behavior of the two constituent materialsreinforcement and matrix. These models are thoroughly described, for example, by TeixeiraDias and Menezes [20].

The constitutive model for the reinforcement is thermoelastic and can thus be described by the following stress tensor evolution relation:

$\dot{\boldsymbol{\sigma}}=2 \mu_{\mathrm{R}} \mathbf{D}^{\mathrm{te}}+\left[\left(k_{\mathrm{R}}-\frac{2}{3} \mu_{\mathrm{R}}\right) \operatorname{tr}\left(\mathbf{D}^{\mathrm{te}}\right)-3 k_{\mathrm{R}} \alpha_{\mathrm{R}} \dot{T}\right] \mathfrak{I}$

where $\mathbf{D}^{\text {te }}=\alpha_{\mathrm{R}} \dot{T} \mathfrak{I}$ is the thermoelastic part of the total strain rate tensor.

The constitutive equations used in the present work for the metallic matrix material have, among others, the big advantage of using a scalar parameter to represent the internal state of the material. This parameter models the resistance of the material to plastic flow. The behavior law for the matrix material is described in the present work by the following stress rate evolution function:

$$
\begin{aligned}
\dot{\boldsymbol{\sigma}}= & 2 \mu_{\mathrm{M}}\left(\mathbf{D}-\mathbf{D}^{\mathrm{vp}}\right) \\
& +\left[\left(k_{\mathrm{M}}-\frac{2}{3} \mu_{\mathrm{M}}\right) \operatorname{tr}(\mathbf{D})-3 k_{\mathrm{M}} \alpha_{\mathrm{M}} \dot{T}\right] \mathfrak{I} .
\end{aligned}
$$

$\mathbf{D}$ is the total strain rate tensor and its viscoplastic part, $\mathbf{D}^{\mathrm{vp}}$, can be calculated as [21]
$\mathbf{D}^{\mathrm{vp}}=\frac{3 \dot{\bar{\varepsilon}}^{\mathrm{p}}}{2 \bar{\sigma}} \boldsymbol{\sigma}^{\prime}$

In this expression $\dot{\bar{\varepsilon}}^{p}$ is the equivalent plastic strain rate which is a function of the equivalent stress $\bar{\sigma}$, the internal scalar parameter $s$ and the absolute temperature $T$, i.e.

$$
\begin{aligned}
\dot{\bar{\varepsilon}}^{\mathrm{p}} & =f(\bar{\sigma}, s, T) \\
& =A \exp \left(-\frac{Q}{R_{\mathrm{g}} T}\right)\left[\sinh \left(\xi \frac{\bar{\sigma}}{s}\right)\right]^{1 / m},
\end{aligned}
$$

$A, Q, m$ and $\xi$ are material parameters. The internal state variable $s$ is defined by

$\dot{s}=f(\bar{\sigma}, s, T) h(\bar{\sigma}, s, T)=\dot{\bar{\varepsilon}}^{\mathrm{p}} h$,

where $h$ can be associated, for example, to the hardening and dynamic restoration effects. This kind of constitutive equations are very stable in numerical terms $[22,23]$ which facilitates their implementation into numerical simulation codes.

\section{Numerical integration}

It is widely recognized that the physical notion of rate-independent plastic deformation is not more than a convenient approximation to the reality and only valid for limited temperature ranges. Plastic deformation is obviously rate dependent, even at low temperatures [24].

As is often the case with physical processes of identical complexity to the ones presented in the present work, the differential equations described in previous sections can only be integrated numerically. There are several possible integration methods, namely: (i) totally explicit or forward; (ii) totally implicit or regressive and (iii) semi-implicit or forward gradient methods.

The constitutive equations used are known to be numerically stable. Nevertheless, using an explicit integration scheme it becomes necessary to adopt very small time increments in order to guarantee the numerical stability of the integration process $[11,24,25]$.

It is possible to implement a semi-implicit integration method in order to avoid the problems inherent to the totally explicit or totally implicit 
methods. Those methods use a Taylor development of the constitutive functions to approximate the implicit schemes. Significantly larger time increments can be used in the forward gradient methods without numerical stability problems $[1,11,15]$. Thus, the numerical integration of the constitutive models presented in the previous sections is made with a forward gradient algorithm in the form initially proposed by Peirce et al. [26] and afterwards developed by Anand et al. [22], Lush and Anand [25], Teodosiu and Menezes [11] and Teixeira-Dias and Menezes [27].

The main goal of the time integration procedure is to determine the correct values of the state variables in a configuration at time instant $t_{n+1}$, starting with the known values of the same variables in the previous configuration, at time $t_{n}$. For this purpose, the viscoplastic part of the strain rate tensor, $\mathbf{D}^{\mathrm{vp}}$, in the current configuration is approximated using a ponderation factor between its values in configurations $n$ and $n+1$. The value of $\mathbf{D}^{\mathrm{vp}}$ at instant $n+1$ is determined with a first order Taylor approximation around $t=t_{n}$. The plastic strain increment can be calculated from this approximation as

$\Delta \varepsilon^{\mathrm{p}}=\int_{t_{n}}^{t_{n+1}} \mathbf{D}^{\mathrm{vp}} \mathrm{d} t$.

If an explicit integration method was to be used, the plastic strain increment $\Delta \boldsymbol{\varepsilon}^{\mathrm{p}}$ would be calculated as a function of the viscoplastic strain rate tensor at increment $n$ only, i.e.

$\Delta \boldsymbol{\varepsilon}^{\mathrm{p}}=\mathbf{D}_{n}^{\mathrm{vp}} \Delta t$.

In the forward gradient method, this increment is determined with a weighted strain rate tensor, such that,

$\Delta \varepsilon^{\mathrm{p}}=\mathbf{D}_{w}^{\mathrm{vp}} \Delta t$.

In this equation, $\mathbf{D}_{w}^{\mathrm{vp}}$ is the weighted viscoplastic strain rate tensor calculated with the strain rate tensors at the beginning and end of the increment:

$\mathbf{D}_{w}^{\mathrm{vp}}=\mathbf{D}_{n}^{\mathrm{vp}}+\Phi\left(\mathbf{D}_{n+1}^{\mathrm{vp}}-\mathbf{D}_{n}^{\mathrm{vp}}\right)$.

The scalar parameter $\Phi$, defined in the interval $[0 ; 1]$, is the weighting factor for the integration process.
In order to determine an approximation to the tensor $\mathbf{D}_{w}^{\mathrm{vp}}$, and remembering Eq. (18), a first order Taylor approximation is developed around time instant $t_{n}$, i.e.

$\mathbf{D}_{n+1}^{\mathrm{vp}}=\mathbf{D}_{n}^{\mathrm{vp}}+\frac{\partial \mathbf{D}_{n}^{\mathrm{vp}}}{\partial f} \Delta f+\frac{\partial \mathbf{D}_{n}^{\mathrm{vp}}}{\partial \bar{\sigma}} \Delta \overline{\boldsymbol{\sigma}}+\frac{\partial \mathbf{D}_{n}^{\mathrm{vp}}}{\partial \boldsymbol{\sigma}^{\prime}} \Delta \boldsymbol{\sigma}^{\prime}$.

Performing the partial derivatives of $\mathbf{D}_{n}^{\mathrm{vp}}$ results in

$$
\begin{aligned}
\mathbf{D}_{n+1}^{\mathrm{vp}}= & \frac{3 f_{n}}{2 \bar{\sigma}_{n}} \boldsymbol{\sigma}_{n}^{\prime}+\frac{3 \boldsymbol{\sigma}_{n}^{\prime}}{2 \bar{\sigma}_{n}} \Delta f+\frac{3}{2} f_{n} \boldsymbol{\sigma}_{n}^{\prime}\left(-\frac{1}{\bar{\sigma}_{n}^{2}}\right) \Delta \bar{\sigma} \\
& +\frac{3 f_{n}}{2 \bar{\sigma}_{n}} \Delta \boldsymbol{\sigma}^{\prime} .
\end{aligned}
$$

In this equation, the equivalent plastic strain rate increment is

$\Delta f=\Delta \bar{\varepsilon}^{\mathrm{p}}=\frac{\partial f_{n}}{\partial \bar{\sigma}} \Delta \bar{\sigma}+\frac{\partial f_{n}}{\partial s} \Delta s+\frac{\partial f_{n}}{\partial T} \Delta T$.

The equivalent stress increment is

$\Delta \bar{\sigma}=\frac{\partial \bar{\sigma}}{\partial \boldsymbol{\sigma}^{\prime}}: \Delta \boldsymbol{\sigma}^{\prime}$.

Introducing the equivalent stress $\bar{\sigma}$ and differentiating with respect to $\boldsymbol{\sigma}_{n}^{\prime}$, results in

$\frac{\partial \bar{\sigma}}{\partial \boldsymbol{\sigma}^{\prime}}=\frac{1}{2}\left(\frac{3}{2} \boldsymbol{\sigma}_{n}^{\prime}: \boldsymbol{\sigma}_{n}^{\prime}\right)^{-1 / 2}\left(3 \boldsymbol{\sigma}_{n}^{\prime}\right)=\frac{3}{2 \bar{\sigma}_{n}} \boldsymbol{\sigma}_{n}^{\prime}$.

Combining Eqs. (28) and (29) leads to

$\Delta \bar{\sigma}=\frac{3}{2 \bar{\sigma}_{n}} \boldsymbol{\sigma}_{n}^{\prime}: \Delta \boldsymbol{\sigma}^{\prime}$

Inserting the stress rate tensor and integrating over time increment $\Delta t$, the stress increment becomes

$\Delta \boldsymbol{\sigma}^{\prime}=2 \mu_{\mathrm{M}}\left(\Delta \boldsymbol{\varepsilon}^{\prime}-\Delta \boldsymbol{\varepsilon}^{\mathrm{p}}\right)$,

where the total strain increment and its deviatoric part are, respectively,

$\Delta \boldsymbol{\varepsilon}=\int_{t_{n}}^{t_{n}+\Delta t} \mathbf{D} \mathrm{d} t \quad$ and $\quad \Delta \boldsymbol{\varepsilon}^{\prime}=\Delta \boldsymbol{\varepsilon}-\frac{1}{3} \operatorname{tr}(\Delta \boldsymbol{\varepsilon})$.

Introducing the Taylor approximation of $\mathbf{D}_{n+1}^{\mathrm{vp}}$, given by Eq. (26), and substituting the equivalent stress increment and equivalent plastic strain rate, leads to 


$$
\begin{aligned}
\Delta \varepsilon^{\mathrm{p}}= & \left(\frac{3 \boldsymbol{\sigma}_{n}^{\prime}}{2 \bar{\sigma}_{n}}\right)\left[f_{n}+\Phi\left(\frac{\partial f_{n}}{\partial \bar{\sigma}} \Delta \bar{\sigma}+\frac{\partial f_{n}}{\partial s} \Delta s+\frac{\partial f_{n}}{\partial T} \Delta T\right)\right] \Delta t \\
& +\Phi \frac{3 f_{n}}{2 \bar{\sigma}_{n}}\left[\Delta \boldsymbol{\sigma}^{\prime}-\left(\boldsymbol{\sigma}_{n}^{\prime}: \Delta \boldsymbol{\sigma}^{\prime}\right) \frac{3 \boldsymbol{\sigma}_{n}^{\prime}}{2 \bar{\sigma}_{n}^{2}}\right] \Delta t
\end{aligned}
$$

or, in a more compact way,

$$
\Delta \boldsymbol{\varepsilon}^{\mathrm{p}}=\Delta \bar{\varepsilon}^{\mathrm{p}}\left(\frac{3 \boldsymbol{\sigma}_{n}^{\prime}}{2 \bar{\sigma}_{n}}\right)+\frac{3}{2 h_{1}}\left[\Delta \boldsymbol{\sigma}^{\prime}-\left(\boldsymbol{\sigma}_{n}^{\prime}: \Delta \boldsymbol{\sigma}^{\prime}\right) \frac{3 \boldsymbol{\sigma}_{n}^{\prime}}{2 \bar{\sigma}_{n}^{2}}\right] .
$$

In this expression,

$$
h_{1}=\frac{\bar{\sigma}_{n}}{\Phi f_{n} \Delta t},
$$

and the equivalent plastic strain increment is

$$
\Delta \bar{\varepsilon}^{\mathrm{p}}=\left[f_{n}+\Phi\left(\frac{\partial f_{n}}{\partial \bar{\sigma}} \Delta \bar{\sigma}+\frac{\partial f_{n}}{\partial s} \Delta s+\frac{\partial f_{n}}{\partial T} \Delta T\right)\right] \Delta t .
$$

Performing the scalar product of the deviator stress tensor $\boldsymbol{\sigma}_{n}^{\prime}$ by the plastic strain increment $\Delta \varepsilon^{\mathrm{p}}$ and introducing the equivalent stress definition results in

$\boldsymbol{\sigma}_{n}^{\prime}: \Delta \varepsilon^{\mathrm{p}}=\bar{\sigma}_{n} \Delta \bar{\varepsilon}^{\mathrm{p}}$.

Combining Eqs. (30), (31) and (38) leads to

$\Delta \bar{\sigma}=\frac{3 \mu_{\mathrm{M}}}{\bar{\sigma}_{n}} \boldsymbol{\sigma}_{n}^{\prime}: \Delta \boldsymbol{\varepsilon}^{\prime}-3 \mu_{\mathrm{M}} \Delta \bar{\varepsilon}^{\mathrm{p}}$,

where the scalar product $\sigma_{n}^{\prime}: \Delta \varepsilon^{\prime}$ can be simplified replacing $\boldsymbol{\sigma}_{n}^{\prime}$ and $\Delta \boldsymbol{\varepsilon}^{\prime}$ by their definitions, i.e.

$\boldsymbol{\sigma}_{n}^{\prime}: \Delta \boldsymbol{\varepsilon}^{\prime}=\boldsymbol{\sigma}_{n}^{\prime}: \Delta \boldsymbol{\varepsilon}-\frac{1}{3} \operatorname{tr}\left(\boldsymbol{\sigma}_{n}\right) \mathfrak{I}: \Delta \boldsymbol{\varepsilon}$,

that allows Eq. (39) to be written as

$\Delta \bar{\sigma}=\frac{3 \mu_{\mathrm{M}}}{\bar{\sigma}_{n}} \boldsymbol{\sigma}_{n}^{\prime}: \Delta \boldsymbol{\varepsilon}-3 \mu_{\mathrm{M}} \Delta \bar{\varepsilon}^{\mathrm{p}}$.

Returning to Eq. (37), replacing the equivalent stress increment $\Delta \bar{\sigma}$ and recalling that $\Delta s=\Delta \bar{\varepsilon}^{p} h$ leads to

$\Delta \bar{\varepsilon}^{\mathrm{p}}=\frac{\Delta t}{1+v_{n}}\left(f_{n}+\Phi \frac{3 \mu_{\mathrm{M}}}{\bar{\sigma}_{n}} \frac{\partial f_{n}}{\partial \bar{\sigma}} \boldsymbol{\sigma}_{n}^{\prime}: \Delta \boldsymbol{\varepsilon}+\Phi \frac{\partial f_{n}}{\partial T} \Delta T\right)$,

where
$v_{n}=\Phi \frac{\partial f_{n}}{\partial \bar{\sigma}} G_{n} \Delta T$ and

$G_{n}=3 \mu_{\mathrm{M}}-h_{n}\left(\frac{\partial f_{n}}{\partial s} / \frac{\partial f_{n}}{\partial \bar{\sigma}}\right)$.

Relating Eqs. (31) and (35) leads to the following relation for the plastic strain increment:

$$
\begin{gathered}
\Delta \boldsymbol{\varepsilon}^{\mathrm{p}}=\Delta \bar{\varepsilon}^{\mathrm{p}}\left(\frac{3 \boldsymbol{\sigma}_{n}^{\prime}}{2 \bar{\sigma}_{n}}\right)+\frac{3}{2 h_{1}}\left[2 \mu_{\mathrm{M}}\left(\Delta \boldsymbol{\varepsilon}^{\prime}-\Delta \boldsymbol{\varepsilon}^{\mathrm{p}}\right)\right. \\
\left.-\frac{3 \mu_{\mathrm{M}}}{\bar{\sigma}_{n}^{2}}\left(\boldsymbol{\sigma}_{n}^{\prime}: \Delta \boldsymbol{\sigma}^{\prime}\right) \boldsymbol{\sigma}_{n}^{\prime}\right] .
\end{gathered}
$$

Substituting the incremental deviatoric strain tensor, $\Delta \boldsymbol{\varepsilon}^{\prime}$, and reorganizing the terms in the previous equation finally results in

$$
\begin{aligned}
\Delta \boldsymbol{\varepsilon}^{\mathrm{p}}= & \frac{3 \bar{\mu}_{n}}{\mu_{\mathrm{M}}}\left(1+\frac{3 \mu_{\mathrm{M}}}{h_{1}}\right) \frac{\boldsymbol{\sigma}_{n}^{\prime}}{2 \bar{\sigma}_{n}} \Delta \overline{\boldsymbol{\varepsilon}}^{\mathrm{p}}+\frac{3 \bar{\mu}_{n}}{h_{1}} \\
& \times\left[\Delta \boldsymbol{\varepsilon}-\frac{1}{3} \operatorname{tr}(\delta \boldsymbol{\varepsilon})\right]-\frac{9 \bar{\mu}_{n}}{h_{1} \bar{\sigma}_{n}^{2}}\left(\boldsymbol{\sigma}_{n}^{\prime}: \Delta \boldsymbol{\varepsilon}\right) \boldsymbol{\sigma}_{n}^{\prime},
\end{aligned}
$$

where

$\bar{\mu}_{n}=\frac{\mu_{\mathrm{M}}}{1+3 \mu_{\mathrm{M}} / h_{1}}$.

In order to calculate the incremental value of the stress tensor, the behavior law, given by Eq. (17), must be integrated along the time increment $\Delta t=\left[t_{n} ; t_{n+1}\right]$, i.e.

$$
\begin{aligned}
\Delta \boldsymbol{\sigma}= & 2 \mu_{\mathrm{M}}\left(\Delta \boldsymbol{\varepsilon}-\Delta \boldsymbol{\varepsilon}^{\mathrm{p}}\right)+\left(k_{\mathrm{M}}-\frac{2}{3} \mu_{\mathrm{M}}\right) \operatorname{tr}(\Delta \boldsymbol{\varepsilon}) \mathfrak{I} \\
& -3 k_{\mathrm{M}} \alpha_{\mathrm{M}}(\Delta T) \mathfrak{J} .
\end{aligned}
$$

Introducing the plastic strain increment given by Eq. (45) leads to

$$
\begin{aligned}
\Delta \boldsymbol{\sigma}= & 2 \mu_{\mathrm{M}} \Delta \boldsymbol{\varepsilon}+\bar{\lambda}_{n} \operatorname{tr}(\Delta \boldsymbol{\varepsilon}) \mathfrak{I}-K_{1}\left(\boldsymbol{\sigma}_{n}^{\prime}: \Delta \boldsymbol{\varepsilon}\right) \boldsymbol{\sigma}_{n}^{\prime} \\
& -K_{2} \boldsymbol{\sigma}_{n}^{\prime}-3 k_{\mathrm{M}} \alpha_{\mathrm{M}}(\Delta T) \mathfrak{I} .
\end{aligned}
$$

In this relation

$$
\begin{aligned}
K_{1} & =\frac{3}{\bar{\sigma}_{n}^{2}}\left[\frac{v_{n}}{1+v_{n}} \frac{3 \mu_{\mathrm{M}}^{2}}{G_{n}}-\left(\mu_{\mathrm{M}}-\bar{\mu}_{n}\right)\right], \\
K_{2} & =\frac{\Delta t}{1+v_{n}}\left(f_{n}+\Phi \frac{\partial f_{n}}{\partial T} \Delta T\right) \frac{3 \mu_{\mathrm{M}}}{\bar{\sigma}_{n}}, \\
\bar{\lambda}_{n} & =k_{\mathrm{M}}-\frac{2}{3} \bar{\mu}_{n} .
\end{aligned}
$$


Finally, and after some algebraic manipulations

$\Delta \boldsymbol{\sigma}=\mathbf{M}^{\mathrm{sec}}: \Delta \boldsymbol{\varepsilon}-K_{2} \boldsymbol{\sigma}_{n}^{\prime}-3 k_{\mathrm{M}} \alpha_{\mathrm{M}}(\Delta T) \mathfrak{I}$.

In this expression, $\mathbf{M}^{\text {sec }}$ is the elastic-viscoplastic secant modulus, whose components are

$M_{i j k l}^{\mathrm{sec}}=\bar{\lambda}_{n} \delta_{i j} \delta_{k l}+\bar{\mu}_{n}\left(\delta_{i k} \delta_{j l}+\delta_{i l} \delta_{j k}\right)-K_{1} \sigma_{i j}^{\prime} \sigma_{k l}^{\prime}$.

$\delta_{i j}$ is the Kronecker delta.

\subsection{Virtual work principle and discretization}

For the thermomechanical model described above, and considering that only thermal loads will be applied, the virtual work principle can be written in its classical forma as [28]

$\int_{V} \boldsymbol{\sigma} \delta \boldsymbol{\varepsilon} \mathrm{d} V=0 \quad$ with $i, j=1, \ldots, 3$,

where $V$ is the overall volume of the solid body.

The dual-phase material is discretized in hexahedral three-dimensional finite elements with eight nodes, trilinear interpolation functions, eight integration points for complete integration and one for reduced integration. The volumetric part of the displacement gradient is interpolated with a reduced selective scheme $[23,29]$, in order to avoid the development of locking effects.

The finite element discretization of Eq. (54), for a generic time increment $n$, leads to a standard linear algebraic system of equations in the form

$\mathbf{K}_{n} \Delta \mathbf{u}_{n}=\Delta \mathbf{f}_{n}$,

where $\Delta \mathbf{u}_{n}$ is the displacement increment vector, $\mathbf{K}_{n}$ is the global stiffness matrix and $\Delta \mathbf{f}_{n}$ is the incremental nodal force vector, all evaluated at time instant $t_{n}$ and corresponding to the time increment $\Delta t$. The solution of system (55) is then used to update the system configuration and all the state variables.

\subsection{Time increment optimization}

The incremental time integration procedure described above can become inaccurate in situations where the plastic flow rate changes very rapidly [22]. Thus, it is necessary to implement an automatic time-step optimization algorithm in order to account for these changes. The automatic time-step algorithm used is based on a measure of the rate of change of the plastic flow rate tensor and is thoroughly described elsewhere [27]. The optimization algorithm uses two step reduction criteria. The first one is based on a scalar control parameter that represents the maximum absolute value, over all the integration Gauss points of the difference between the equivalent plastic strain rate $\dot{\bar{\varepsilon}}^{p}$ at the end and beginning of the current increment, multiplied by the size of the time increment itself. The second criteria is based on the determination of the maximum value of the increment of the equivalent plastic strain over all the integration points of the structure.

A time-step reduction factor $r$ is calculated after the determination of the two optimization parameters by combining the two criteria. The time increment size is then updated according to

$\Delta t_{\text {new }} \rightsquigarrow \frac{r_{\text {cut }}}{r} \Delta t_{\text {old }}$ or $\Delta t_{\text {new }} \rightsquigarrow r_{\text {enl }} \Delta t_{\text {old }}$,

whether $r>1$, leading to a reduction of the timestep size, or $r<1$, leading to an increase of the time-step size, respectively. $r_{\text {cut }}$ is an optimization reduction factor. The enlargement factor, $r_{\mathrm{enl}} \in[1.1 ; 2.5]$, can be calculated dynamically for each time step according to the expression described below:

$r_{\text {enl }}=2.5-1.4\left\{\frac{\exp \left(F_{\mathrm{c}} \frac{r-0.2}{0.6}\right)-1}{\exp \left(F_{\mathrm{c}}\right)-1}\right\}$,

where $F_{\mathrm{c}}$ is a correction factor defining the shape of the optimization profile [27].

\section{Results and discussion}

In this section, we present the results of a set of numerical simulations. They were performed in order to validate the incremental models proposed in the previous sections. The case study was selected and its results were compared with the results of similar problems solved by other authors.

The case study concerns the simulation of the cooling down process of an aluminum matrix MMC with SiC reinforcement. The initial temperature is $T=220^{\circ} \mathrm{C}$ and the final temperature is $T=20^{\circ} \mathrm{C}$. The cooling rate is $|\dot{T}|=100^{\circ} \mathrm{C} \mathrm{s}^{-1}$. 


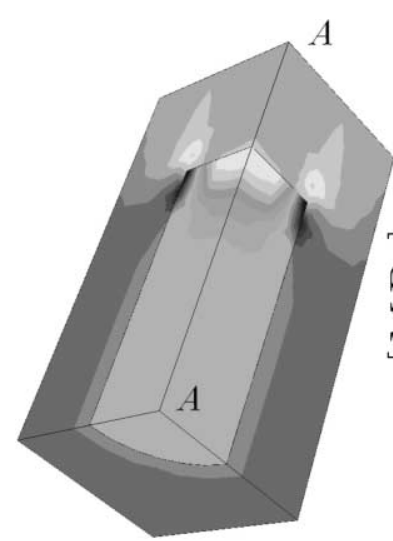

(a)

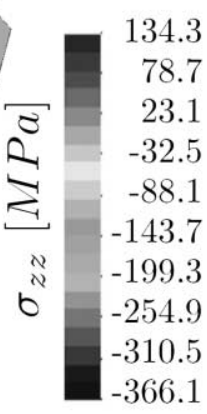

$-366.1$

Fig. 3. Distributions of (a) the $\sigma_{z z}$ stress com
with $11 \%$ volume fraction of reinforcement.

The numerical simulations are performed with a three-dimensional unit cell representative of a long cylindrical fiber reinforced MMC with 11\% volume fraction of reinforcement material.

In Fig. 3(a) and (b) are represented the distributions of the $\sigma_{z z}$ stress component and of the equivalent stress $\bar{\sigma}$, respectively. It can be noticed that, at the top of the unit cell the equivalent stress levels are significantly higher than the yield stress of the matrix material [21]. This fact leads to un-

desirable plastic strain localization inside the matrix of the composite material.

The graphic in Fig. 4 shows the distributions of $\sigma_{z z}$ and $\bar{\sigma}$ along line $A A$, indicated in Fig. 3. These results are compared with the ones obtained by Jain et al. [30]. It can be promptly stated that the results are considerably close to each other. Eventual discrepancies are certainly due to the fact that Jain et al. [30] used an elastic-plastic rate-independent constitutive model for the matrix material.

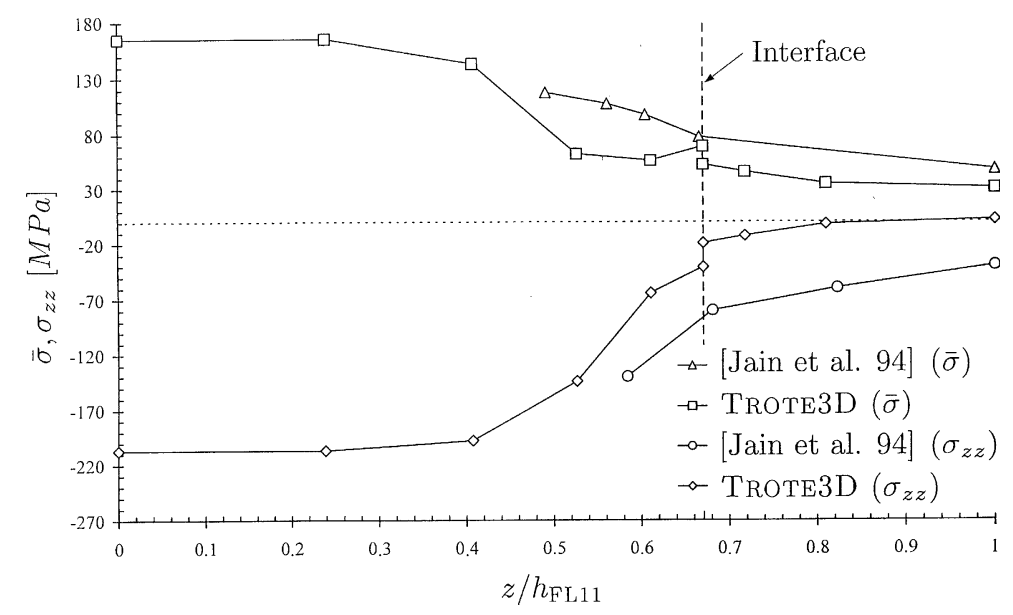

Fig. 4. Evolution of residual stresses $\bar{\sigma}$ and $\sigma_{z z}$ for case study 1, of height $h_{\mathrm{FL} 11}=\overline{A A}$. Also plotted are the results obtained by [30] with a similar model. 


\section{Conclusions}

A three-dimensional incremental micromechanical model applicable to the study of the micromechanical behavior of dual-phase materials with distinct constitutive behaviors was presented. The kinematic model is based on the decomposition of the transformation gradient in elastic and plastic parts.

The constitutive models used to test the incremental model proposed correspond to an $\mathrm{Al}-\mathrm{SiC}$ $\mathrm{MMC}$ and consider the reinforcement component to have a thermoelastic behavior and the matrix material to behave in a thermoelastic-viscoplastic way. The increments of the relevant state variables are determined for each of the materials using a semi-implicit forward gradient time integration procedure.

The model was applied in a finite element method algorithm and tested with numerical examples in order to evaluate its capabilities. The numerical results were compared with similar problems solved with different methods by other authors. Residual stress fields were determined in an $\mathrm{Al}-\mathrm{SiC} \mathrm{MMC}$ material with volume fractions of reinforcement material of $11 \%$. The geometrical model used represents a cylindrical fiber reinforced MMC.

It was stated that the levels of equivalent stress can reach values above the yield limit, leading to the development of undesired plastic strain close to the matrix/reinforcement interface.

Very good accordance was obtained with the results presented by Jain et al. [30]. Thus, the numerical methods and algorithms presented proved to be very efficient and reliable when predicting the micromechanical behavior of dual-phase materials with very distinct constitutive behaviors.

\section{References}

[1] L. Anand, Int. J. Plast. 1 (1985) 213.

[2] G.L. Povirk et al., Mater. Sci. Eng. A 125 (1990) 129.

[3] G.L. Povirk et al., Mater. Sci. Eng. A 132 (1991) 31.

[4] Y.-L. Shen et al., Metall. Trans. A 25A (1994) 839.

[5] L.L. Shaw, D.B. Miracle, Acta Mater. 44 (5) (1996) 2043.

[6] P.J. Ward et al., Acta Mater. 44 (5) (1996) 1717.

[7] J.C. Michel, P. Suquet, Model. Simul. Mater. Sci. Eng. 2 (1994) 637.

[8] N. Ramakrishnan, Acta Mater. 44 (1) (1996) 69.

[9] D.B. Zahl et al., Model. Simul. Mater. Sci. Eng. 2 (1994) 267.

[10] M. Suéry et al., Mater. Sci. Eng. A 167 (1993) 97.

[11] C. Teodosiu, L.F. Menezes, Rev. Roum. Sci. Techn. - Méc. Appl. 36 (3/4) (1991) 243

[12] R. Kolhe et al., Acta Mater. 44 (1) (1996) 279.

[13] E. Zywicz, D.M. Parks, Comp. Sci. Tech. 33 (1988) 295.

[14] M. Vedula et al., Composites 19 (2) (1988) 133.

[15] H.M. Ledbetter, M.W. Austin, Mater. Sci. Eng. 89 (1987) 53.

[16] S. Ho, A. Saigal, Mater. Sci. Eng. A 183 (1994) 39.

[17] R.J. Arsenault, M. Taya, Acta Metall. 35 (3) (1987) 651.

[18] P.J. Withers et al., Acta Metall. 37 (11) (1989) 3061.

[19] M.L. Dunn, M. Taya, J. Mater. Sci. 29 (1994) 2053.

[20] F. Teixeira-Dias, L.F. Menezes, Comp. Mater. Sci. 21 (1) (2001) 26.

[21] S.B. Brown et al., Int. J. Plast. 5 (1989) 95.

[22] L. Anand et al., Research report, Department of Mechanical Engineering, MIT, 1985.

[23] T.J.R. Hughes, Int. J. Num. Meth. Eng. 15 (1980) 1413.

[24] A. Lush et al., Int. J. Plast. 5 (1989) 521.

[25] A. Lush, L. Anand, in: Proc. NUMIFORM'86, 1986, p. 131.

[26] D. Peirce et al., Comp. Struct. 18 (1984) 875.

[27] F. Teixeira-Dias, L.F. Menezes, Int. J. Num. Meth. Eng. 50 (3) (2001) 629.

[28] O.C. Zienkiewicz, R.L. Taylor, The Finite Element Method, vol. 1, fourth ed., McGraw-Hill Book Company Europe, Berkshire, England, 1994, p. 225.

[29] H. Shimodarira, Int. J. Num. Meth. Eng. 21 (1985) 89.

[30] M. Jain et al., Mater. Sci. Eng. A 183 (1994) 111. 